\title{
Information and Data Processing in the Scheme of $1 \rightarrow 3$ Optimal Phase-covariant Quantum Cloning Machine
}

\author{
LiZhi $\mathrm{Yu}^{1,2}$ \\ 1. School of Physics and Electrics, Fuyang Teachers College, Fuyang 236029, China \\ 2. Fuyang vocational college of science and technology, Fuyang, Anhui 236000, China \\ ylz0706@163.com
}

\begin{abstract}
Keywords: data processing, quantum cloning, phase-covariant
Abstract. Quantum cloning plays the key role in information and data processing. We proposed a scheme for implementing the optical $1 \rightarrow 3$ phase covariant cloning, which optimally clones qubits belonging to the equatorial plane of the Bloch sphere. We show how the $1 \rightarrow 3$ phase covariant cloning can be implemented by a smart modification of the standard universal quantum machine by a projection of the output states over the symmetric subspace.
\end{abstract}

\section{Introduction}

In recent years, a great deal of efforts has been devoted to the realization of the optimal approximations to the quantum cloning and flipping operations over an unknown qubit. Entanglement attracted much attention in information and data processing, for example, teleportation ${ }^{[1]}$, cryptography ${ }^{[2,3]}$, cloning ${ }^{[4,}$ ${ }^{5]}$, etc. Recently, the quantum cloning has been researched widely. Owing to no-cloning theorem ${ }^{[6,7]}$, a quantum state can't be produced precisely. Buzek and Hillery ${ }^{[8]}$ first discussed this possibility, which is particularly important to quantum communication and cryptography. They showed that it was possible to create copies (approximate clones) of unknown quantum states with a quality that did not depend on the initial state. Many people began to make study to clone quantum states under all these limitations, that is to say, the approximate cloning of quantum input states without knowing their information completely in advance.

Not only the perfect cloning of unknown qubit is forbidden but also perfect cloning of subsets containing non orthogonal states. This no-go theorem ensures the security of cryptographic protocols as BB84. Recently state dependent cloning machines have been investigated that are optimal respect to a given ensemble [12]. Quantum cloning can be divided into three types in terms of different input states for discrete quantum systems. When the information of the input pure state is completely unknown, this is the so called optimal universal quantum cloning (UQC) ${ }^{[9-15]}$. If the modules of the complex coefficients of the input state are known but their phases are unknown, this is the so-called optimal phase-covariant cloning (PCC). If the phases of the complex coefficients of the input state are known but their modules are unknown, this is the so-called optimal real state cloning (RSC). According to the difference in fidelity among these clones, three cloners can be divided into symmetric and asymmetric cloners. Thus, realizing these cloning processes is meaningful.

Recently, a lot of schemes have been proposed to implement various cloning ${ }^{[16-17]}$, and some experimental realizations have also been reported. The partial a-priori knowledge of the state allows reaching a higher fidelity than for the universal cloning. In particular the $\mathrm{N} \rightarrow \mathrm{M}$ phase-covariant quantum cloning machine (PQCM) considers the cloning of $\mathrm{N}$ into $\mathrm{M}$ output qubits, where the input ones belong to the equatorial plane of the corresponding Poincare' sphere. In the present article we will restrict ourselves to the case in which $\mathrm{N}=1$. For $\mathrm{M}$ assuming odd values it is found $f_{\mathrm{cov}}^{1 \rightarrow M}=\frac{1}{4}\left(3+M^{-1}\right)$ while in the case of even M-values $f_{\text {cov }}^{1 \rightarrow M}=\frac{1}{2}\left(3+\frac{1}{2} \sqrt{1+2 M^{-1}}\right)$. In the present work we report the implementation of a $1 \rightarrow$ 3PQCM by adopting a modified standard $1 \rightarrow 2$ UQCM and by further projecting the output qubits over the symmetric subspace. 


\section{Implementing the Optical $1 \rightarrow 3$ Cloning}

Let the state of the input qubit be expressed by

$$
|\phi\rangle_{s}=\alpha|0\rangle_{s}+\beta|1\rangle_{s}
$$

where $|a|^{2}+|b|^{2}=1$. The output state of the $1 \rightarrow 2$ UQCM device reads

$$
|\Sigma\rangle_{S A B}=\sqrt{\frac{2}{3}}|\phi\rangle_{S}|\phi\rangle_{A}\left|\phi^{\times}\right\rangle_{B}-\frac{1}{\sqrt{6}}\left(|\phi\rangle_{S}\left|\phi^{\times}\right\rangle_{A}+\left|\phi^{\times}\right\rangle_{S}|\phi\rangle_{A}\right)|\phi\rangle_{B}
$$

The qubits $\mathrm{S}$ and $\mathrm{A}$ are the optimal cloned qubits while the qubit $\mathrm{B}$ is the optimally flipped one. We perform the operation $U_{B}=\sigma_{y}$ on the qubit B. We will get

$$
\begin{aligned}
& |\Re\rangle_{S A B}=\left(\mathrm{I}_{S} \otimes \mathrm{I}_{A} \otimes \mathrm{I}_{B}\right)|\Sigma\rangle_{S A B} \\
& =\sqrt{\frac{2}{3}}|\phi\rangle_{S}|\phi\rangle_{A}\left|\phi^{\times}\right\rangle_{B}-\frac{1}{\sqrt{6}}\left(|\phi\rangle_{S}\left|\phi^{\times}\right\rangle_{A}+\left|\phi^{\times}\right\rangle_{S}|\phi\rangle_{A}\right)|\phi\rangle_{B},
\end{aligned}
$$

By this non-universal cloning process three asymmetric copies have been obtained: two clones (qubits $\mathrm{S}$ and $\mathrm{A}$ ) with fidelity $5 / 6$, and a third one (qubit B) with fidelity $2 / 3$. We may now project $\mathrm{S}, \mathrm{A}$ and $\mathrm{B}$ over the symmetric subspace and obtain three symmetric clones with a higher average fidelity. The symmetric subspace has dimension 4 since three qubits are involved. The probability of success of the 3 projection is equal to $8 / 9$. The normalized output state is

$$
|\zeta\rangle_{S A B}=\frac{\sqrt{3}}{2}|\phi\rangle_{S}|\phi\rangle_{A}|\phi\rangle_{B}-\frac{1}{2 \sqrt{3}}\left(|\phi\rangle_{S}\left|\phi^{\times}\right\rangle_{A}\left|\phi^{\times}\right\rangle_{B}+\left|\phi^{\times}\right\rangle_{S}|\phi\rangle_{A}\left|\phi^{\times}\right\rangle_{B}+\left|\phi^{\times}\right\rangle_{S}\left|\phi^{\times}\right\rangle_{A}|\phi\rangle_{B}\right) \text {. }
$$

Let us now estimate the output density matrices of the qubits $\mathrm{S}, \mathrm{A}$ and $\mathrm{B}$

$$
\rho_{S}=\rho_{A}=\rho_{B}=\frac{5}{6}|\phi\rangle\left\langle\phi\left|+\frac{1}{6}\right| \phi^{\times}\right\rangle\left\langle\phi^{\times}\right|,
$$

This leads to the fidelity is $5 / 6$ equal to the optical one.

By applying a different unitary operator UB to the qubit B we can implement the phase covariant cloning for different equatorial planes. Interestingly, note that by this symmetrization technique a depolarizing channel on channel B transforms immediately the non-universal phase covariant cloning into the universal $1 \rightarrow 3 \mathrm{UQCM}$ with the overall fidelity $7 / 9$. This represents a relevant new proposal to be implemented within the $1 \rightarrow 2$ UQCM QI-OPA devices or other $1 \rightarrow 2$ cloning schemes. Let us return to the $1 \rightarrow 3 \mathrm{PQCM}$. In the present scheme the input qubit to be injected into a QI-OPA over the spatial mode $\mathrm{k}_{1}$ with wavelength. We have

$$
|\phi\rangle_{\text {in }}=\alpha|H\rangle+\beta|V\rangle
$$

Where $|H\rangle$ and $|V\rangle$ stand for horizontal and vertical polarization.

Let us consider the injected photon in the mode $\mathrm{k} 1$ to have any linear polarization $\pi=\phi . \mathrm{We}$ express this $\pi$ state as

$$
\dot{a}_{1 \phi}^{\dagger}|0,0\rangle_{k_{1}}=|1,0\rangle_{k_{1}}
$$

Where $|m, n\rangle_{k_{1}}$ represents a product state with $\mathrm{m}$ photons of the mode $\mathrm{k} 1 \mathrm{with}$ polarization $\phi$, and $\mathrm{n}$ photons with polarization $\phi^{\times}$. The same reason for the mode $\mathrm{k} 2$ to have any linear polarization $\pi=\phi$.

The $U_{B}=\sigma_{y}$ flipping operation on the output mode $\mathrm{k} 2$, implemented by means of two wave-plates, transformed the QI-OPA output state into

$$
|\Re\rangle_{S A B}=\sqrt{\frac{2}{3}}|2,0\rangle_{k_{1}}|1,0\rangle_{k_{2}}-\sqrt{\frac{1}{3}}|1,1\rangle_{k_{1}}|0,1\rangle_{k_{2}},
$$

The physical implementation of the projector on the three photons $\dot{\pi}$ state was carried out by linearly superimposing the modes $\mathrm{k} 1$ and $\mathrm{k} 2$ on the $50: 50$ beam-splitter BSA and then by selecting the case in which the 3 photons emerged from BSA on the same output mode $\mathrm{k} 3$ (or, alternatively on $\mathrm{k} 4$ ). By adopting the previous relations and by considering the case in which 3 photons emerge over the mode $\mathrm{k} 3$, the output state is found to be 


$$
\frac{1}{2 \sqrt{2}}\left(a_{3 \phi}^{\dagger 3}+a_{3 \phi}\right)|0,0\rangle_{k_{3}}=\frac{\sqrt{3}}{2}|3,0\rangle_{k_{3}}+\frac{1}{2}|1,2\rangle_{k_{3}},
$$

The output fidelity is $5 / 6$.

Interestingly, the same overall state evolution can also be obtained, with no need of the final BSA summarization, at the output of a QI-OPA with a type II crystal working in a collinear configuration, as proposed by. In this case the interaction Hamiltonian

$$
H_{\text {coll }}=i x \mathrm{~h}\left(\$_{H} \$ \mathbb{B}_{V}\right)+\text { h.c. }
$$

acts on a single spatial mode $\mathrm{k}$. A fundamental physical property of consists of its rotational invariance under U(1) transformations, that is, under any arbitrary rotation around the z-axis. Indeed eq.(10) can be re-expressed as

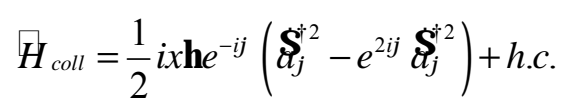

Let us consider an injected single photon with state

$$
|\varphi\rangle_{\text {in }}=\frac{1}{\sqrt{2}}\left(|H\rangle+e^{i \phi}|V\rangle\right)
$$

The first contribution to the amplified state is identical to the output state obtained with the device dealt with in the present work up to a phase factor which does not affect the fidelity value.

The UV pump beam with back reflected by the spherical mirror Mp with $100 \%$ reflectivity and $\mu$-adjustable position Z, excited the NL crystal in both directions and, i.e. correspondingly oriented towards the right hand side and the left hand side of Fig.1. A Spontaneous Parametric Down Conversion (SPDC) process excited by the UV mode created singlet-states of photon polarization. The photon of each SPDC pair emitted over the mode was back-reflected by a spherical mirror M into the NL crystal and provided the $\mathrm{N}=1$ quantum injection into the OPA excited by the UV beam associated with the back-reflected mode. The twin SPDC photon emitted over mode, selected by the "state analyzer" consisting of the combination (Wave-Plate + Polarizing Beam Splitter: WPT + PBST ) and detected by DT , provided the "trigger" of the overall conditional experiment. Because of the EPR non-locality of the emitted singlet, the selection made on implied deterministically the selection of the input state -iinon the injection mode.

The output state on mode was analyzed by the setup shown in the inset the field on mode was disregarded, for simplicity. The polarization state on mode was analyzed by the combination of the wp WPC and of the polarizer beam splitter PBSC. The different overall quantum efficiencies have been taken intoaccount in the processing of the experimental data. The precise sequence of the experimental procedures was suggested by the following considerations.

In the scheme, both the optical swithes $S_{1}$ and $S_{2}$ are closed at the begining, and the cavity and fiber modes are in the vacuum state. The quantum information is encoded on the state of atom 1 , the initial input state for three atoms. For the sake of completeness, we have carried out an experiment setting the pump mirror in the position and changing the position. The injected state was $|\varphi\rangle_{\text {in }}=|+\rangle$.

Due to quantum interference, the coincidence rate was enhanced by a factor moving from the position to the condition. These results are a further demonstration of the 3-photon interference in the Hong-Ou-Mandel device.

\section{Conclusion}

In conclusion, we have implemented the optimal quantum tri-plicators for equatorial qubits. The present approach can be extended in a straightforward way to the case of $1 \rightarrow$ M PQCM for M odd. The results are relevant in the modern science of quantum communication as the PQCM is deeply connected to the optimal eavesdropping attack at BB84 protocol, which exploits the transmission of quantum states belonging to the $\mathrm{x}-\mathrm{z}$ plane of the Bloch sphere. The optimal fidelities achievable for 
equatorial qubits are equal to the ones considered for the four states adopted in BB84 [13]. In addition, the phase covariant cloning can be useful to optimally perform different quantum computation tasks adopting qubits belonging to the equatorial subspace.

\section{Acknowledgements}

This work was financially supported by The Talent Foundation of Anhui Provincial Higher Education ( No. 2013SQRL135ZD ) .

\section{References}

[1] C.H. Bennett, G.Brassard, C. Crepeau, et al., Phys.Rev.Lett.70 (1993) 1895.

[2] C.H. Bennett, S.J.Wiesner, Phys.Rev.Lett.69 (1992) 2881.

[3] A.K. Ekert, Phys.Rev.Lett. 67 (1991) 661.

[4]L.M. Duan, G.C. Guo, Phys. Rev. Lett. 80 (1998) 4999.

[5] M. Murao, D. Jonathan, M.B. Plenio, et al., Phys. Rev. A 59 (1999) 156.

[6] W.K. Wootters, W.H. Zurek, Nature (London) 299 (1982) 802.

[7] D. Dieks, Phys. Lett. A 92 (1982) 271.

[8] V. Buzek, M. Hillery, Phys. Rev. A 54 (1996) 1844.

[9] L.M. Duan, G.C. Guo, Phys. Rev. Lett. 80 (1998) 4999.

[10] A. K. Pati, Phys. Rev. A 61 (2000) 022308.

[11] S.B.Zhen, G.C.Guo, Phys.Rev. A 63(2011):044302.

[12] W. Zhang, J. Liu, et al., Chin. Phys. B 17 (2008) 3203.

[13] L.H.Yan, Y.F.Gao, H.J.Wang, et al., J. At, Mol. Phys. 26 (2009):875.

[14] H. J. Briegel, R. Raussendorf, Phys. Rev. Lett. 86 (2001) 910.

[15] P.Dong, Z.Y. Xue, M. Yang, et al., Phys.Rev.A 73 (2006) 033818.

[16] D. C. Li, Z. L. Cao, Commun. Theor. Phys.47 464(2007).

[17] A. Furusawa, J.L. Sorensen, S.L. Braunstein et al. Science. 282 (1998) 706. 